\title{
Çocukluk Taş Hastalığında Extracorporeal Shock Wave Lıthotrıpsy: Hala Yaşıyor ve Çok Etkili
}

\author{
Yılmaz Aksoy ${ }^{1}$, Turgut Yapanoğlu²
}

Atatürk Üniversitesi, Tıp Fakültesi, Çocuk Ürolojisi Bilim Dalı ve Üroloji Anabilim Dalı ${ }^{1}$ Erzurum

Giriş

Sok dalga litotripsi (SWL) ilk defa Almanya'da 7 Şubat ל̧1980 yılında klinik kullanıma girmiştir (1). Bu tarihten sonra yaygın şekilde kullanılan bu yeni yöntem, ancak 6 yıl sonra Newman ve ark.'ları tarafından çocuklarda uygulanarak yayımlanmıştır (2). Bu gecikmenin asıl nedeni, gelişen bir organizmada SWL'nin böbreklerde veya karaciğer, over ve pankreas gibi komşu organlarda oluşturabileceği hasarın bilinmiyor olmasıdır. Yapılan deneysel ve klinik çalışmalar ile birlikte uzun dönem takiplerde; böbrekte oluşan hasarın minimal olduğu ve önemli skara ve hipertansiyona yol açmadığı bildirilmiştir (3-5). İlk pediatrik SWL serisini diğer çalışmalar takip etmiş ve 2000'li yıllarda olgu sayıları yüksek seriler yayımlanmıştır (6-8). Erişkinlerle karşılaştırıldığında SWL'nin daha düşük kilovolt $(\mathrm{kV})$, şok sayısı ve seans sayısı ile çocuklarda benzer başarı oranlarına sahip olduğu bildirilmiştir (9). Çocuklarda uygulanan enerjinin $14-22 \mathrm{kV}$, seans başına şok sayısının 1800-2000 olması (5 yaş altı çocuklarda ise seans başına 1000 şok dalgasını geçmemesi), en fazla 3 seans uygulanması ve böbreklerde iki seans arasında en az 15 gün geçmesi önerilmektedir (10). SWL'nin başarısında çocuğun yaşı, taş boyutu, taşın lokalizasyonu, kaliks anatomisi, taşın cinsi, konjenital anomali varlığ törün tipi rol oynar (10). İnfantlarda SWL başarısı oldukça yüksek olup, Shukla ve ark.'ları çalışmalarında \%100 başarı bildirmişlerdir (11). İnfant ve küçük çocuklarda SWL'nin çok başarılı olmasının sebebi olarak; taş oluşumunun kısa sürede gerçekleşmiş olması, cilt-taş mesafesinin kısa olması ve bu nedenle şok dalgalarının vücut tarafından az absorbe edilmesi gösterilmektedir. Bunun yanında üreterin kısa ve kompliansının iyi olması ve çocukların daha erken ve etkin mobilize olabilmeleri de bu açıdan önemli faktörlerdir (11). Taş boyutu 10 mm'den küçük olduğunda SWL başarısı ortalama \%90'larda iken; 20 mm'den büyük taşlarda başarı \%60'lar kadardır (Tablo 1).

Tablo 1: Taş boyutuna göre SWL başarısı

\begin{tabular}{|c|c|c|c|c|}
\hline \multirow[t]{2}{*}{ Otör } & \multirow[t]{2}{*}{ Lokalizasyon } & \multicolumn{3}{|c|}{ Taş boyutuna göre taşsızlık oranları } \\
\hline & & $<10 \mathrm{~mm}$ & $10-20 \mathrm{~mm}$ & $20 \mathrm{~mm}<$ \\
\hline Slavkovic ve ark. ${ }^{12}$ & $\begin{array}{l}\text { Böbrek } \\
\text { Üreter }\end{array}$ & $\begin{array}{l}\% 95.8 \\
\% 97.1\end{array}$ & $\begin{array}{c}\% 77.3 \\
\% 80\end{array}$ & $\begin{array}{c}\% 66.7 \\
\% 50\end{array}$ \\
\hline Aksoy ve ark. ${ }^{7}$ & $\begin{array}{l}\text { Böbrek } \\
\text { Üreter }\end{array}$ & $\begin{array}{l}\% 100 \\
\% 100\end{array}$ & $\begin{array}{l}\% 93.5 \\
\% 66.6\end{array}$ & $\begin{array}{c}\% 66.6 \\
-\end{array}$ \\
\hline Müslümanoğlu ve ark. ${ }^{6}$ & $\begin{array}{l}\text { Pelvis } \\
\text { Kaliks }\end{array}$ & $\begin{array}{l}\% 92.3 \\
\% 62.5\end{array}$ & $\begin{array}{c}\% 68.3 \\
\% 40\end{array}$ & $\begin{array}{c}\% 50 \\
\% 42.9\end{array}$ \\
\hline Badawy ve ark. ${ }^{8}$ & $\begin{array}{l}\text { Böbrek } \\
\text { Üreter }\end{array}$ & $\begin{array}{l}\% 93.8 \\
\% 91.2\end{array}$ & $\begin{array}{l}\% 85.8 \\
\% 43.7\end{array}$ & - \\
\hline
\end{tabular}

Tablo 2: Üreter taşlarında SWL'nin başarısı

\begin{tabular}{|c|c|c|c|c|}
\hline Otör & $\begin{array}{l}\text { Olgu sayısı } \\
\text { (n) }\end{array}$ & $\begin{array}{c}\text { Proksimalüreter } \\
\text { SFR (\%) }\end{array}$ & $\begin{array}{l}\text { Orta üreter } \\
\text { SFR (\%) }\end{array}$ & $\begin{array}{l}\text { Distalüreter } \\
\text { SFR (\%) }\end{array}$ \\
\hline Al-Busaidy ve ark. ${ }^{14}$ & 63 & 92 & 89 & 85 \\
\hline Landau ve ark. ${ }^{15}$ & 38 & 95 & 100 & 100 \\
\hline Abdel-Khalek ve ark. ${ }^{16}$ & $938^{*}$ & 92.8 & 85.4 & 82.7 \\
\hline Müslümanoğlu ve ark. ${ }^{17}$ & 192 & 94 & 94 & 89 \\
\hline Lu ve ark. ${ }^{18}$ & 115 & 96.2 & 87.5 & 95.7 \\
\hline Badawy ve ark. ${ }^{8}$ & 50 & 76.5 & 80 & 72.3 \\
\hline
\end{tabular}

*:Erişkin ve çocuklardan oluşan bir seri, SFR:Stone-free rate 
Pelvis renalis, orta ve üst pol böbrek taşları ile proksimal üreter taşlarında SWL başarısı yüksek, alt pol ve distal üreter taşlarında ise düşük bulunmuştur (13). Proksimal üreter taşlarında SWL'nin başarısı \%90'lar civarındadır. Distal üreter taşlarında ise; SWL başarısı taşı odaklamadaki teknik zorluklardan dolayı daha düşüktür (Tablo 2).

İnfundibulum boynunun dar olması, infundibulum uzunluğunun $>3 \mathrm{~cm}$ olması, infundibulopelvik açının $<70$ derece olması ve kompleks kaliks anatomisi varlığ 1 taşsızlık oranlarını olumsuz olarak etkilemektedir (19). Alt kaliks taşlarında kırılan taşın düşürülememesi nedeniyle SWL'nin başarısı \%50 ile \%62'lere kadar düşmektedir (13). Taşın cinsi de; SWL'nin başarısını etkileyen diğer bir faktördür. Struvit ve kalsiyum apatit taşları SWL ile kolay parçalanırken; sistin taşları ve kalsiyum okzalatmonohidrat taşları SWL'ye dirençli taşlardır (13). SWL öncesi taşın kimyasal yapısı bilinmediğinden SWL için taşın uygun olup olmadığına nonkontrast bilgisayarlı tomografi (NKBT)' de taşın HU dansitesi ölçülerek karar verilebilir. Şayet taşın HU dansitesi 1000'in üzerinde ise SWL'ye dirençli olduğu düşünülmelidir (20). Renal anomalili çocuklarda SWL başarısının düşük olması, taşın odaklanmasındaki ve pasajındaki zorluklardan dolayıdır (13). Litotriptör tipinin de; stone-free oranlarında rolü olduğu bilinmektedir. Elektrohidrolik jeneratörlü makinelerin başarısı daha yüksek, elektromanyetik ve piezoelektrik güç kaynağ kullanan makinelerin etkinliği ise daha düşüktür. Ancak yeni jenerasyon makinelerin fokus alanları dar olduğundan, başarıları biraz düşük olmakla birlikte, komplikasyonları da bununla orantılı olarak daha azdır. Diğer bir sorun eski makinelerdeki şok başlığını barındıran su yastığının büyük olması nedeniyle özellikle çocuk üreter taşlarının odaklanmasındaki zorluklardır $(10,21)$. Farklı makinelerin kullanılmasıyla yapılmış karşılaştırmalı çalışmalar literatürde az sayıda mevcuttur (Tablo 3). masalara adapte edilebilmektedirler (25). Endoüroloji'deki hızlı değişime ayak uydurma adına; SWL teknolojisinde de gelişme ve arayışlar sürmektedir. Bilgisayar yardımlı odaklama ve navigasyon, fokus alanında değişiklikler, 3-boyutlu taş görüntüleme sistemi, dalga basınçlarının optimizasyonu, kombine şok dalga kaynaklarının birlikte kullanılması ve eş zamanlı ikiz atım tekniği bunlardan bazılarıdır (25).

European Society for Paediatric Urology (ESPU) 2015 kılavuzunda SWL tedavisi $10 \mathrm{~mm}$ 'den küçük pelvis renalis taşlarında kanıt seviyesi 1A düzeyinde önerilirken, 10-20 $\mathrm{mm}$ pelvis renalis taşları ve $10 \mathrm{~mm}$ 'den küçük alt kaliks taşlarında 2B kanıt düzeyinde ilk basamak tedavi seçeneği olarak önerilmektedir (13). D'Addessi ve ark.'ları pediatrik SWL uygulanmış 21 çalışmayı gözden geçirdiğinde, komplikasyon oranının \%18 olduğunu gözlemlemiş ve bu komplikasyonların çoğunun hematüri, ciltte ekimoz, renal kolik, üriner enfeksiyon (UTI) gibi hafif komplikasyonlar olduğunu bildirmiştir (26). Ancak nadiren subkapsüler, intrarenal, perirenal hematom, sepsis ve hemoptizi gibi önemli komplikasyonlar da görülebilmektedir $(7,26)$. Soliter böbrekte taşı olan, taş boyutu $\geq 2 \mathrm{~cm}$ olan ve non-opak taşı olan çocuklarda SWL uygulamadan önce JJ stent konulması önerilmektedir (7). Bununla birlikte bir derlemede JJ stentin üreter peristaltizmini bozduğu, obstrüksiyon yaparak taştan arınmayı zorlaştırdığı, perforasyon, üreteral ve/veya üretral darlık yapabildiği bildirilmektedir (26). Bunun yanında ikinci bir anestezi ile alınması gerektiği ve stente bağlı mesane semptomlarına sebep olarak morbiditeyi artırdığı için kullanılması önerilmemektedir (26). Ancak stent konulmasının taş yolu, obstrüksiyona bağlı pyelonefrit ve sepsis gibi major komplikasyon oranlarını azalttığı da; bir gerçektir (7).

SWL'deki önemli sorunlardan birisi de; $\% 20$ ile $\% 64$ 'lere kadar ulaşan yüksek yeniden tedavi oranlarıdır $(13,21,26)$. $\mathrm{Bu}$ durum hem anestezi sayısını, hem de böbrekte oluşa-

Tablo 3: Farklı litotriptörlerin başarısını karşılaştıran çalışmalar

\begin{tabular}{lccccc}
\hline $\begin{array}{l}\text { Otör } \\
\text { Litotriptör }\end{array}$ & \multicolumn{2}{c}{$\begin{array}{c}\text { Elsobky ve ark. }{ }^{\mathbf{2 2}} \mathbf{( 2 0 0 0 )} \\
\text { MFL 5000 }\end{array}$} & Echolith & Raza ve ark. ${ }^{\mathbf{2 3}}$ (2009) & \multicolumn{2}{c}{$\begin{array}{c}\text { Aksoy ve ark. }{ }^{21} \text { (2009) } \\
\text { MPL 9000 }\end{array}$} & Lithostar & MPL 9000 & Lithostar \\
\hline Hasta (n) & 102 & 40 & 50 & 40 & 173 \\
Stone-free (\%) & 85 & 87 & 95 & 77 & 80 \\
Komplikasyon & $0 / 102$ & $1 / 40$ & $8 / 50$ & $4 / 40$ & 86.2 \\
Re-treatment/hasta & 1.8 & 2.3 & 1.7 & 1.6 & $16 / 173$ \\
\hline
\end{tabular}

İlk makinelerden Dornier HM3 etkinlik olarak hala altın standart olarak kabul edilmektedir (10). Litotriptörlerin etkinliği en iyi modifiye etkinlik değeri (EQmod) kullanılarak hesaplanmaktadır (24). bilecek hasarı artırmaktadır. Çocuklarda SWL uygulamaları sırasında anestezi gereksinimi de; başka bir sorundur. Ancak son yıllarda geliştirilen fokus alanı daha dar olan litotriptörlerle genel anestezi gerekmeksizin sadece sedo-

Taştan arınmış hasta \% - Küratif yardımcı tedaviler \%

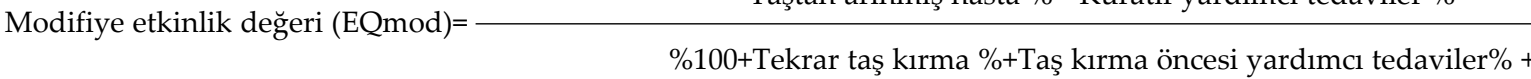

Taş kırma sonrası adjuvan tedaviler \%

Litotriptörlerde elektrohidrolik, elektromanyetik ve piezoelektrik jeneratörler kullanılmıştır. Ancak günümüzde en çok elektromanyetik jeneratörler tercih edilmektedir. Çünkü elektromanyetik jeneratörler daha küçük başlıklarda yeterli şok dalga basıncı sağlayabilmekte, şok dalgaları daha kontrollü ve etkin olmaktadır. Bunun yanında elektrodu daha uzun ömürlü olup, odaklama alanının boyutu değiştirilebilmekte ve kolaylıkla çok fonksiyonlu modüler analjezi yapılarak SWL etkin şekilde kullanılabilmektedir $(21,27)$. Yeni jenerasyon makinelerde SWL infantlarda bile sedo-analjezi ile başarılı bir şekilde uygulanmaktadır. Bir çalışmada Adanur ve ark.'ları infantlarda sedo-analjezi için pentothalsodium 3-4 mg/kg ve fentanyl 1-2 $\mu \mathrm{g} / \mathrm{kg}$ dozlarda kullanıldığını bildirmişlerdir (27). Daha büyük çocuklarda ise; (<5 yaş) kliniğimizde propofol $1-2 \mathrm{mg} / \mathrm{kg}$ ve fentanyl $1-2 \mu \mathrm{g} / \mathrm{kg}$ kombinasyonu kullanılmaktadır (21). Erişkinlerde 
kullanılan klinik olarak önemsiz rezidüel fragman teriminin çocuklarda SWL sonrası $3 \mathrm{~mm}$ ve daha küçük taş fragmanları için kullanılması uygun görülmemiştir. Çünkü bu fragmanların olguların yaklaşık 1/3'ünde büyüdüğü ve yaklaşık yarısında semptom verdiği gösterilmiştir $(28,29)$. SWL sırasında taşın odaklanmasında floroskopi kullanılması diğer bir istenmeyen durumdur. Günümüzde dijital floroskopi sistemlerinin kullanılması düşük radyasyon dozlarında daha kaliteli görüntüler elde edilmesini sağlamıştır. Ayrıca böbrek yerleşimli taşlarda ultrasonografik (US) odaklama da; azımsanmayacak oranlarda başarıyla kullanılmaktadır $(7,21)$. SWL tedavisi sirasında alınan iyonize radyasyon dozu böbrek başına ortalama $6.8 \mathrm{mSv}$ olarak hesaplanmıştır (30). Taşı olan çocuklarda tanıda ve takiplerde kullanılacak görüntüleme yöntemlerinin seçimi de önem arz etmektedir. Başlangıç görüntüleme yöntemi US ve/veya direkt üriner sistem grafisi (DÜS) olmalıdır; çünkü tahmin edilen radyasyon dozu $0.7 \mathrm{mSv}$ 'dir (31). NKBT tanının açık olmadı $\breve{g}_{1}$ veya tedavi planlamasında anatomik detay gerekliliğinde yaptırılmalıdır; çünkü maruz kalınan radyasyon dozu single detector NKBT'de $6.5 \mathrm{mSv}$, multidetector NKBT'de $8.6 \mathrm{mSv}$ ve düşük doz NKBT'de 1.5 mSv olarak hesaplanmıştır. İşte bu nedenle çocuklarda NKBT gerektiğinde ALARA (as low as reasonably achievable) prensibine göre mutlaka düşük doz protokol tercih edilmelidir (30). Yukarıda basedilen tüm bu nedenlerle SWL'nin, erişkinlerde uygulanması 1984 yılında U.S. Food and Drug Administration (FDA) tarafından onaylanmasına rağmen, çocuklarda uygulanmasına dair halen bu kurumun onayı bulunmamaktadır.

Sonuç olarak, Endoürolojik ekipmanlardaki teknolojik ilerlemeler sonucunda daha nazik aletlerin ve daha iyi görüntüleme sistemlerinin geliştirilmesi ile holmium: YAG lazer gibi etkin ve güvenilir intrakorporeal taş kırma yöntemlerinin bulunması endoskopik taş cerrahisini erişkinlerde oldukça popüler hale getirmiştir. Ancak endoürolojik taş cerrahisi çocuk uretra ve üreterlerinin çok nazik olması nedeniyle çocuklarda erişkinlerdeki kadar yaygın kullanım alanı bulamamıştır. SWL çocuk taş hastalığında hala birçok endikasyonda birinci basamak tedavi olmaya devam etmektedir.

\section{Kaynaklar}

1. Chaussy C, Brendel W, Schmiedt E. Extracorporeally induced destruction of kidney Stones by shock waves. Lancet. 1980;2:1265-8.

2. Newman DM, Coury T, Lingeman JE, Mertz JH, Mosbaugh PG, Steele RE, Knapp PM.Extracorporeal shock wave lithotripsy experience in children. J Urol. 1986;136:238-40.

3. Lottmann HB, Archambaud F, Hellal B, Pageyral BM, Cendron M. 99mTechnetium-dimercapto-succinic acidrenalscan in theevaluation of potential long-term renal parenchymal damage associated with extracorporeal shock wave lithotripsy in children. J Urol 1998;159:521-4.

4. Kaji DM, Xie HW, Hardy BE, Sherrod A, Huffman JL. The effects of extracorporeal shock wave lithotripsy on renal growth, function and arterial blood pressure in an animal model. J Urol. 1991;146:544-7.

5. El-Nahas AR, Awad BA, El-Assmy AM, Abou El-Ghar ME, Eraky I, El-Kenawy MR, Sheir KZ. Are there longterm effects of extracorporeal shock wave lithotripsy in paediatric patients? BJU Int 2013;111:666-71.
6. Muslumanoglu AY, Tefekli A, Sarilar O, Binbay M, Altunrende F, Ozkuvanci U. Extracorporeal shock wave lithotripsy as first line treatment alternative for urinary tract stones in children: a large scale retrospective analysis. J Urol. 2003;170:2405-8.

7. Aksoy Y, Ozbey I, Atmaca AF, Polat O. Extracorporeal shock wave lithotripsy in children: experience using a mpl-9000 lithotriptor. World J Urol. 2004;22:115-9.

8. Badawy AA, Saleem MD, Abolyosr A, Aldahshoury M, Elbadry MS, Abdalla MA, Abuzeid AM. Extracorporeal shock wave lithotripsy as first line treatment for urinary tract stones in children: outcome of 500 cases. Int Urol Nephrol. 2012;44:661-6.

9. Kurien A, Symons S, Manohar T, Desai M. Extracorporeal shock wave lithotripsy in children: equivalent clearance rates to adults is achieved with fewer and lower energy shock waves. BJU Int. 2009;103:81-4.

10. Landau EH. Modern Stone management in children. European Urology Supplements 2015;14:12-19.

11. Shukla AR, Hoover DL, Homsy YL, Perlman S, Schurman $S$, Reisman EM. Urolithiasis in the low birth weight infant: the role andefficacy of extracorporeal shock wave lithotripsy. J Urol 2001;165:2320-3.

12. Slavkovic A, Radovanovic M, Vlajkovic M, Novakovic D, Djordjevic N, Stefanovic V. Extracorporeal shock wave lithotripsy in the management of pediatric urolithiasis. Urol Res. 2006;34:315-20.

13. Tekgül S, Dogan HS, Erdem E, Hoebeke P, Ko cvara R, Nijman JM, Radmayr C, Silay MS, SteinR, Undre S. Urinary Stone Disease Guidelines on Paediatric Urology, 2015;51-58.

14. Al Busaidy SS, Prem AR, Medhat M, Giriraj D, Gopakumar P, Bhat HS. Paediatric ureteric calculi: efficacy of primary in situ extra-corporeal shock wave lithotripsy. Br J Urol. 1998;82:90-6.

15. Landau EH, Gofrit ON, Shapiro A, Meretyk S, Katz G, Shenfeld OZ, Golijanin D, Pode D. Extracorporeal shock wave lithotripsy is highly effective for ureteral calculi in children. J Urol. 2001;165:2316-9.

16. Abdel-Khalek M, Sheir K, Elsobky E, Showkey S, Kenawy M. Prognostic factors for extracorporeal shock-wave lithotripsy of ureteric stones--a multivariate analysis study. Scand J UrolNephrol. 2003;37:413-8.

17. Muslumanoglu AY, Tefekli AH, Altunrende F, Karadag MA, Baykal M, Akcay M. Efficacy of extracorporeal shock wave lithotripsy for ureteric stones in children. Int Urol Nephrol. 2006;38:225-9.

18. Lu J, Sun X, He L, Wang Y. Efficacy of extracorporeal shock wave lithotripsy for ureteral stones in children. Pediatr Surg Int. 2009;25:1109-12.

19. Elbahnasy AM, Clayman RV, Shalhav AL, Hoenig DM, Chandhoke P, Lingeman JE, et al. Lower-pole caliceal Stone clearance after shock wave lithotripsy, percutaneous nephrolithotomy, and flexible ureteroscopy: impact of radiographic spatial anatomy. J Endourol. 1998;12:113-9.

20. El-Assmy A, Abou-el-Ghar ME, el-Nahas AR, Refaie HF, Sheir KZ. Multidetector computed tomography: role in determination of urinary Stones composition and disintegration with extracorporeal shock wave lithotripsy--an in vitro study. Urology. 2011;77:286-90.

21. Aksoy Y, Ziypak T, Yapanoglu T. Comparison of the effectiveness and safety of MPL 9000 and Lithostar Modularis shock wave lithotriptors: treatment results of 263 children. Urol Res 2009;37:111-6. 
22. Elsobky E, Sheir K, Madbouly K, Mokhtar AA. Extracorporeal shock wave lithotripsy in children: experience using two-second-generation lithotripters. BJU Int. 2000;86:851-6.

23. Raza SJ, Ather MH. Doesthetype of lithotripter affect outcomes in children with upper tract urolithiasis? J Endourol. 2009;23:223-7.

24. Preminger GM, Clayman R. The changing face of lithotripsy: impact of secondgenerationmachines. In: Proceedings of the 7th World Congress on Endourology and ESWL, Kyoto, Japan, 1989; p.187.

25. Rassweiler JJ, Knoll T, Köhrmann KU, McAteer JA, Lingeman JE, Cleveland RO, Bailey MR, Chaussy C. Shock wave technology andapplication: an update. EurUrol. 2011; 59: 784-96.

26. D'Addessi A, Bongiovanni L, Sasso F, Gulino G, Falabella R, Bassi P. Extracorporeal shock wave lithotripsy in pediatrics. J Endourol. 2008;22:1-12.

27. Adanur S, Ziypak T, Yilmaz AH, Kocakgol H, Aksoy M, Yapanoglu T, Polat O, Aksoy Y. Extracorporeal shock wave lithotripsy under sedoanalgesia fo rtreatment of kidney stones in infants: a single-center experienc ewith 102 cases. Int Urol Nephrol. 2014;46:2095-101.
28. Dincel N, Resorlu B, Unsal A, Tepeler A, Silay MS, Armağan A, Diri A, Sancaktutar AA, Ziypak T, Mir S. Are small residual Stone fragments really insignificant in children? J Pediatr Surg. 2013;48:840-4.

29. El-Assmy A, El-Nahas AR, Harraz AM, El Demerdash Y, Elsaadany MM, El-Halwagy S, Sheir KZ. Clinically insignificant residual fragments: Is it an appropriate term in children? Urology. 2015;86:593-8.

30. John BS, Patel U, Anson K. What radiation exposure can a patient expect during a single Stone episode? J Endourol. 2008;22:419-22.

31. Katz SI, Saluja S, Brink JA, Forman HP. Radiation dose associated with unenhanced CT for suspected renal colic: impact of repetitive studies. AJR Am J Roentgenol. 2006;186:1120-4.

Yazışma Adresi:

Yilmaz Aksoy,

Atatürk Üniversitesi, Tıp Fakültesi, Çocuk Ürolojisi Bilim Dalı, Erzurum

Tel: +905333241693

e-mail:aksoyyilmaz@yahoo.com 